ORIGINAL ARTICLE

\title{
Effect of dry needling of gluteal muscles on straight leg raise: a randomised, placebo controlled, double blind trial
}

\author{
L Huguenin, P D Brukner, P McCrory, P Smith, H Wajswelner, K Bennell
}

Br J Sports Med 2005;39:84-90. doi: 10.1136/bjsm.2003.009431

See end of article for authors' affiliations

Correspondence to: Associate Professor Brukner, Centre for Health, Exercise and Sports Medicine, School of Physiotherapy, University of Melbourne, Melbourne, Victoria 3010, Australia; p.brukner@unimelb. edu.au

Accepted 20 January 2004

\begin{abstract}
Objectives: To use a randomised, double blind, placebo controlled trial to establish the effect on straight leg raise, hip internal rotation, and muscle pain of dry needling treatment to the gluteal muscles in athletes with posterior thigh pain referred from gluteal trigger points.

Methods: A randomised, double blind, placebo controlled trial of 59 male runners was performed during the 2002 Australian Rules football season. Subjects were thoroughly screened and had magnetic resonance imaging of their hamstring muscles to exclude local pathology. The inclusion criterion was reproduction of recognisable posterior thigh pain with the application of digital pressure to the gluteal trigger points. Subjects randomly received either therapeutic or placebo needle treatment on one occasion at their gluteal trigger points. Range of motion and visual analogue scale data were collected immediately before, immediately after, 24 hours after, and 72 hours after the intervention. Range of motion was measured with passive straight leg raise and hip internal rotation. Visual analogue scales were completed for hamstring and gluteal pain and tightness at rest and during a running task.

Results: Magnetic resonance imaging scans revealed normal hamstring musculature in most subjects. Straight leg raise and hip internal rotation remained unchanged in both groups at all times. Visual analogue scale assessment of hamstring pain and tightness and gluteal tightness after running showed improvements immediately after the intervention in both groups $(p=0.001)$, which were maintained at 24 and 72 hours. The magnitude of this improvement was the same for therapeutic and placebo interventions. Resting muscle pain and tightness were unaffected.

Conclusions: Neither dry needling nor placebo needling of the gluteal muscles resulted in any change in straight leg raise or hip internal rotation. Both interventions resulted in subjective improvement in activity related muscle pain and tightness. Despite being commonly used clinical tests in this situation, straight leg raise and hip internal rotation are not likely to help the therapist assess response to treatment. Patient reports of response to such treatment are better indicators of its success. The mechanisms by which these responses occur and the reasons for the success of the placebo needling treatment are areas for further investigation.
\end{abstract}

linically, posterior thigh pain is a common presentation in participants of Australian Rules football and other running sports. This may be a local or a referred phenomenon. ${ }^{1}$ Many patients continue to participate despite reporting subjective performance limitations. In some cases, this pain is most closely reproduced by the application of digital pressure to gluteal muscle trigger points (L Huguenin, unpublished work). This referral of pain to the hamstrings has previously been described in patients with myofascial pain. $^{23}$

Trigger points are localised areas of tenderness within a taut band of muscle fibres. ${ }^{2}$ They are considered a secondary phenomenon to overuse, poor muscle balance, postural abnormalities, or injury. ${ }^{2}$ Active trigger points produce symptoms and pain referral. Latent trigger points are responsible for muscle tightness which is usually asymptomatic but detectable on manual clinical assessment. The diagnosis of an active trigger point relies on the reproduction of recognisable local or referred symptoms on application of digital pressure. An active trigger point also exhibits reproduction of symptoms and a local twitch response to needle penetration. ${ }^{2}$

The mechanism of pain referral from trigger points is controversial. Current evidence points to neurochemical changes at the spinal cord, ${ }^{4-11}$ and sympathetic activation increases this pain perception. ${ }^{12}{ }^{13}$

Trigger points in the gluteal muscles may form therefore as a result of lower back pathology (mild disc disease, lumbar stiffness), sacroiliac joint dysfunction, muscle weakness, or other biomechanical abnormalities of the lower limbs. In athletes, these factors may be related to high training loads, concurrent injuries, fatigue at the end of games, or intrinsic biomechanical imbalances.

The differential diagnosis for referred posterior thigh pain includes structures of the lower back (discs, facet joints), sacroiliac joint, or lumbosacral radiculopathy. ${ }^{1}$ The clinical features of these syndromes are distinct from the syndrome produced by trigger points. Neurological impairment, pain with lumbar movement, and reproduction of pain with manoeuvres other than trigger point palpation are not indicative of trigger point related pain.

Straight leg raise (SLR) and hip internal rotation (hip IR) are commonly used to assess pain in the pelvis and posterior thigh. ${ }^{14}$ Many clinicians use these tests to monitor response to treatment of gluteal trigger points. This has not been validated, although measures change with other therapeutic interventions around the pelvis. ${ }^{15-20}$

Treatment of trigger points in athletes has not previously been described. Treatments investigated in other populations include spray and stretch, ${ }^{2}$ TENS, ${ }^{21}{ }^{22}$ ultrasound, ${ }^{23}$ laser, ${ }^{24-28}$ injection of local anaesthetic, ${ }^{29-31}$ toxic substance, ${ }^{32}{ }^{33}$ or botulinum toxin, ${ }^{34}{ }^{35}$ and dry needling. ${ }^{313637}$ Of the noninvasive treatments, there is no convincing evidence that

Abbreviations: IR, internal rotation; $M R I$, magnetic resonance imaging; SLR, straight leg raise; VAS, visual analogue scale 
Table 1 Findings from magnetic resonance imaging scan of hamstrings of subjects with posterior thigh pain referred from gluteal trigger points

\begin{tabular}{lll}
\hline Location & Findings & Number \\
\hline Intervention leg & $\begin{array}{l}\text { Normal hamstrings } \\
\text { Mild hamstring muscle strain }\end{array}$ & 50 \\
& $\begin{array}{l}\text { Hamstring muscle tear } \\
\text { Mild enthesopathy of hamstring } \\
\text { origin }\end{array}$ & 3 (2 excluded) \\
& Did not attend for appointment & 1 \\
& Total & 61 \\
Non-intervention & Hamstring pathology (tear/ & 5 \\
leg & strain/enthesopathy) & 4 \\
Incidental findings & Quadriceps pathology & 3 \\
& Gluteal muscle pathology & 3 \\
& Osteitis pubis & 2 \\
& Hip joint pathology & 2 \\
\hline & Enchondroma of femur & \\
\hline
\end{tabular}

there is benefit over placebo, with subjective improvements far outweighing objective measures.

Injections have been used for many decades. ${ }^{38-40}$ Improvements in pain are common, but few researchers include a placebo. Injection of local anaesthetic has not been convincingly shown to be superior to injection of normal saline. ${ }^{27}{ }^{41}$

Dry needling has been investigated, and some studies have included a placebo intervention. ${ }^{36} 37$ The placebos have involved subcutaneous penetration. Overall, an equivalent improvement in subjective outcome ratings has been found, without change in objective measures. Subcutaneous injection has previously been postulated to have an effect on pain perception because of gating of local pain impulses from trigger points. ${ }^{42}$

A placebo needle has been described and found to be reliable for use in blinded acupuncture research. ${ }^{43}$ The needle had a blunt tip and did not pierce the skin. By telescoping into its handle, this needle accounted for visual cues, which are not relevant for treatment of the back or gluteal region.

The purpose of this study was to evaluate the effect of both therapeutic and placebo dry needling on SLR, hip IR, muscle pain, and muscle tightness in athletes with trigger point related posterior thigh pain.

\section{METHODS}

\section{Participants}

Fifty nine male athletes were recruited from football clubs, advertisements, flyers, and referral from private clinics. Sample size was calculated for $80 \%$ power, using an assumed

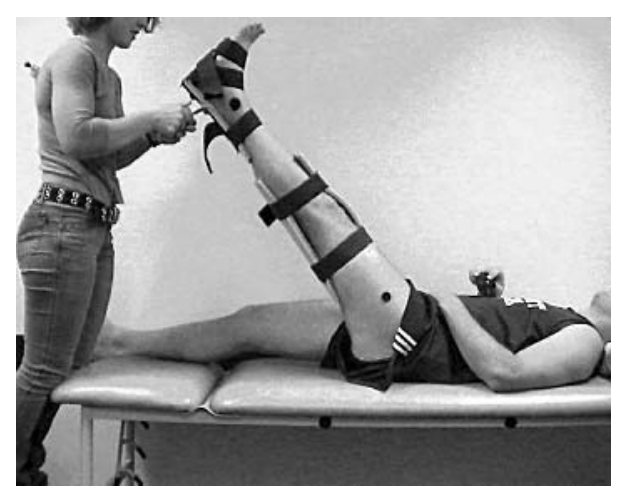

Figure 1 Subject positioning and marker placement for measurement of straight leg raise. The subject is holding the remote control unit in his right hand.

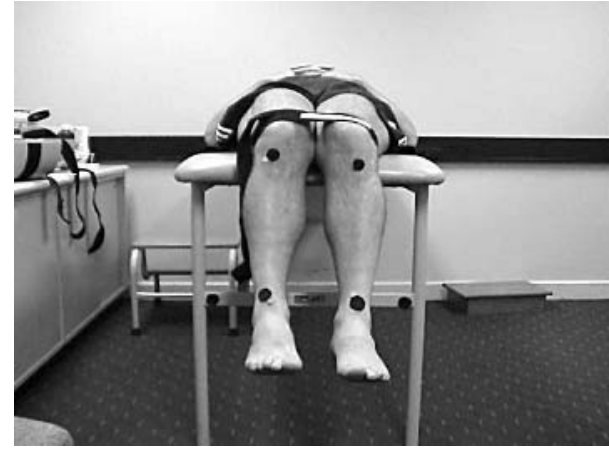

Figure 2 Resting position for the measurement of hip internal rotation.

change in SLR of $15^{\circ}$ and visual analogue scale (VAS) of $2 \mathrm{~cm}$, based on clinical experience, as no published data were available. Subjects completed a screening questionnaire and had a physical examination. Those accepted into the study had a gradual onset of hamstring pain, reproduction of recognisable hamstring pain with pressure on their gluteal trigger points, a good understanding of written and spoken English, and were able to attend all sessions.

Reasons for exclusion were a history of hamstring tear within the previous six weeks, clinical evidence of a hamstring tear, magnetic resonance imaging (MRI) evidence of a hamstring tear, significant lower back injury within six weeks, clinical evidence of significant lumbar or sacroiliac joint contribution to their pain, clinical evidence of a radiculopathy or neurological impairment, needle phobia, bleeding disorder, anticoagulant medication, previous experience with dry needling for myofascial pain, or an inability to reproduce symptoms with trigger point palpation. All subjects had an MRI scan of their hamstrings.

Testing was delayed for an intercurrent illness or injury that prevented completion of the running task, or if the MRI scan exhibited extremely mild non-localised hamstring oedema. This is an extremely mild strain injury and correlates with full strength and symptom free return to sport within 7-14 days. ${ }^{44}$ Subjects describing long term hamstring pain, but exhibiting mild oedematous changes on MRI consistent with a mild new injury, were reassessed at one to two weeks and were only included if their hamstring was not tender and if they had full resisted strength on manual testing and continued to complain of symptoms consistent with those of the long term complaint. It was essential that their symptoms be reproducible with gluteal trigger point pressure. Table 1

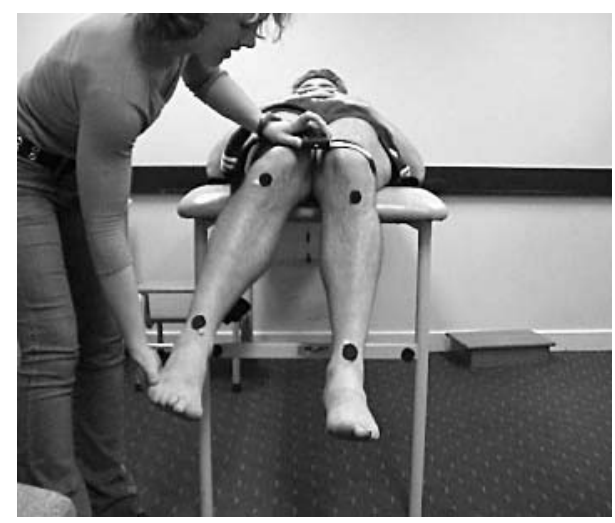

Figure 3 Measurement of hip internal rotation. The remote control is held by the examiner's left hand and the hip is passively internally rotated. The technique is reversed for analysis of the left leg. 
gives the MRI findings; "strain" describes these very mild injuries. Two hamstring tears were excluded. One tear seen on MRI was included because it was seen as resolving oedema in the distal medial hamstring from a recognised injury. The subject had no tenderness in the area and had full strength. He was able to differentiate his usual, long standing pain from that suffered with this acute injury. His usual pain persisted after resolution of the distal medial pain and was reproduced with trigger point pressure. He was therefore included in the trial.

\section{Ethical consideration}

Ethical approval was obtained from the University of Melbourne human research ethics committee. All subjects provided written informed consent.

\section{Procedure}

All outcome measures were collected by the same, blinded observer (LH) before, and immediately, 24 hours, and 72 hours after the intervention.

\section{Straight leg raise}

Subjects were given clear verbal and written instructions before being positioned supine on an examination couch and instructed to remain relaxed. Their upper arms were by their sides, with head resting comfortably on one pillow, and contralateral leg resting on the couch. Knee and ankle splints were used to standardise the position of the intervention leg. Splints were made of moulded thermoplastic, padded, and fixed to the limb with Velcro straps. The contralateral limb was not stabilised in any way, nor was the pelvis, as research to date has not shown any improvement in SLR reliability by these measures. ${ }^{15}{ }^{16}$ Subjects were reminded to maintain contact between the contralateral limb and couch throughout.

A $2.5 \mathrm{~cm}$ black marker was placed $10 \mathrm{~cm}$ above the lateral malleolus in the line of the fibula. A second marker was placed two thirds of the distance between the knee and the greater trochanter, in the mid lateral line of the thigh. Reference markers were permanently attached to the horizontal rail of the couch and not moved during the trial.

The leg was gradually lifted by the examiner at the heel splint with a hand held force transducer. Whenever the lifting force decreased as the result of active hip flexion, the subject was reminded to remain relaxed. The subject notified the examiner at two separate points. Pl was defined as the first onset of sensation (stretch or pain) in the posterior thigh (hamstrings). P2 was defined as the subject's perceived maximum range of motion. This was usually indicated by the maximum tolerable hamstring discomfort. Should that point not have been reached, but the contralateral leg or pelvis began lifting off the couch, it was felt that the maximum isolated SLR range had been achieved and this was classed as tP2.

The subject indicated each of these points verbally, at which time the examiner immediately stopped moving the leg, and the subject took a still digital photograph by pressing a button on a remote control unit (fig 1). The remote control unit minimised any stretch effect by reducing the time in any one position.

Measures were repeated three times. The contralateral leg was measured before the intervention only.

\section{Hip internal rotation}

Subjects were supine and relaxed, knees flexed passively to $90^{\circ}$ at the edge of the couch (fig 2). The head rested on one pillow, with arms relaxed. A Velcro fastener secured the knees together to avoid abduction/adduction. A black marker was placed on both legs at the most prominent point of the tibial tuberosity. Another marker was placed midway between the malleoli on the anterior ankle joint line. Reference markers were permanently placed on the horizontal rail of the couch.

The examiner used a remote control to photograph the resting position of the legs. The heel of the right leg was passively moved laterally, internally rotating the hip with gentle downwards pressure on the thigh by the examiner to prevent hip flexion (fig 3). When the hip was unable to be rotated further (end of range), the investigator took a remote photograph. The left leg was similarly assessed, and each repeated up to a total of three measurements. Although active range of motion has been used in previous studies, ${ }^{45}$ passive range more closely represents common clinical practice.

\section{Analysis of range of motion}

Range of motion was recorded by a Sony digital handycam model DCR-TRVZOE and stored on a 64 MB Sony Memory Stick. Camera position was $2 \mathrm{~m}$ from the edge of the couch, on a tripod. Tripod legs were shortened one third (fixed

\begin{tabular}{|c|c|c|c|c|}
\hline & \multicolumn{4}{|c|}{ Range of motion $\left(0^{\circ}\right)$} \\
\hline & \multicolumn{2}{|c|}{ Intervention leg } & \multicolumn{2}{|c|}{ Control leg } \\
\hline & $\begin{array}{l}\text { Placebo } \\
(n=30)\end{array}$ & $\begin{array}{l}\text { Real } \\
(n=29)\end{array}$ & $\begin{array}{l}\text { Placebo } \\
(n=30)\end{array}$ & $\begin{array}{l}\text { Real } \\
(n=29)\end{array}$ \\
\hline \multicolumn{5}{|l|}{ SLR to $P 1$} \\
\hline Before & $46(10)$ & $53(13)$ & $49(10)$ & $54(12)$ \\
\hline After & $50(11)$ & $55(13)$ & - & - \\
\hline 24 hours after & 47 (11) & 52 (12) & - & - \\
\hline 72 hours after & 48 (12) & 55 (11) & - & - \\
\hline \multicolumn{5}{|l|}{ SLR to $P 2$} \\
\hline Before & 73 (14) & $72(14)$ & $69(14)$ & 71 (13) \\
\hline After & $73(16)$ & $72(14)$ & - & - \\
\hline 24 hours affer & 71 (17) & 71 (14) & - & - \\
\hline 72 hours after & $72(17)$ & $73(15)$ & - & - \\
\hline \multicolumn{5}{|l|}{ Hip IR } \\
\hline Before & $30(6)$ & $31(9)$ & $28(6)$ & $29(8)$ \\
\hline After & $32(8)$ & $31(8)$ & - & - \\
\hline 24 hours affer & 31 (7) & $31(8)$ & - & - \\
\hline 72 hours affer & 31 (7) & 31 (8) & - & - \\
\hline
\end{tabular}



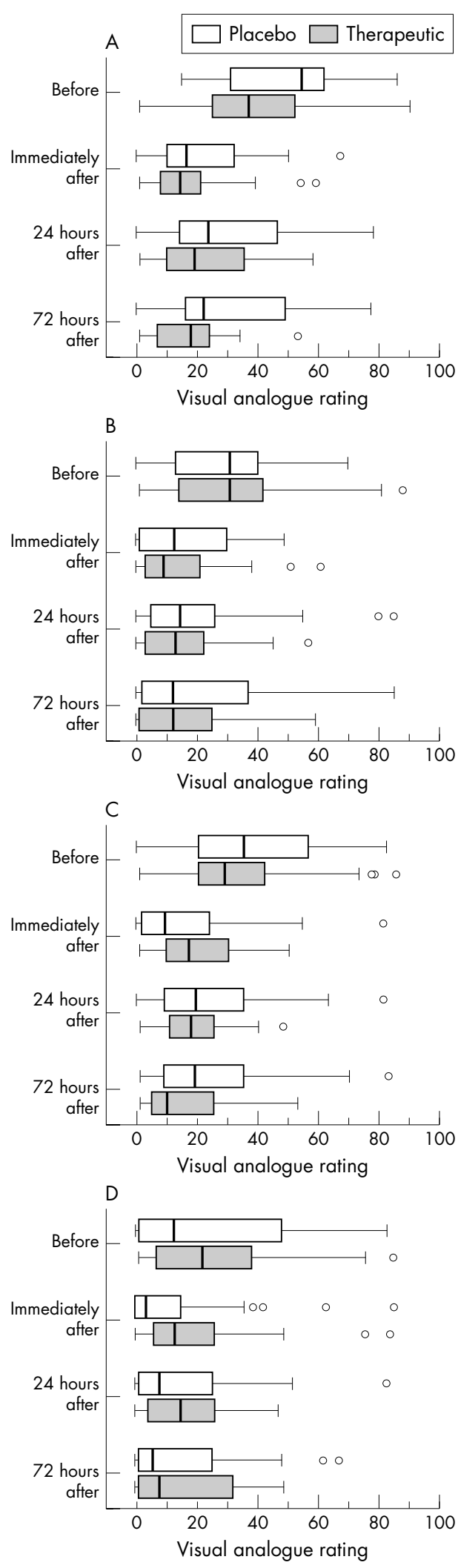

Figure 4 Effects of dry needling on hamstring tightness (A) and pain (B) and gluteal tightness (C) and pain (D) after running. The median is represented by a line in the box, and the limits of the box are the interquartile distance. The whiskers extend to the minimum and maximum values, and outliers are represented by small circles. settings) for hip IR measurement to reduce parallax error. Camera and couch positions were standardised by permanent marks on the floor. Photographs of known angles revealed an error of approximately $0.5^{\circ}$ at this camera distance.

Digital photographic data were analysed on an Apple Macintosh computer using Igor Image Analyse 2.5. Angles were calculated relative to the horizontal alignment of the bed and are not true values of range of motion. However, as the study is designed to assess any change relative to baseline, it is more important that the measure is repeatable than that it is a pure measure of range. SLR was calculated as the angle between the leg (along the line of the two markers) and the horizontal rail of the couch (fig 1). Hip IR angles were calculated by subtracting the resting position from the end of range. They therefore represented total internal rotatory excursion of the hip. This made allowance for subjects with anteverted femoral necks or other hip joint restrictions that may have given them a resting angle of less than $0^{\circ}$ from the vertical and a total range that would appear to be close to zero if measured from the vertical. Results were manually entered on recording sheets and transposed into a Microsoft Excel data base.

\section{Measurement error}

Range of motion photographs were reanalysed for a randomly selected sample of 20 subjects. The mean difference between the first and second analysis was $0.29^{\circ}$, with a standard deviation of $0.19^{\circ}$.

\section{Reliability analysis}

Reliability testing was performed for SLR and hip IR before the start of the study. These results are available from the authors on request. Intraclass correlation coefficients for SLR to Pl were 0.76 for the right leg and 0.89 for the left leg. Intraclass correlation coefficients were 0.92 and 0.95 for SLR to P2 and 0.80 and 0.83 for hip IR. This corresponded to a $95 \%$ confidence interval of $5^{\circ}$ to $7^{\circ}$ for each measurement, which was considered acceptable.

\section{Visual analogue scales}

Pain and tightness in both the hamstrings and gluteals were measured on four unmarked $10 \mathrm{~cm}$ VASs. The right hand end of the scale represented the worst pain or tightness ever experienced by the subject. Pain was defined as "muscle pain, such as that you may have experienced with a tear or a cork" (haematoma). Tightness was defined as a "lack of give, or loss of range" of the muscle. Verbal and written instructions were given. Subjects placed a mark on the scale for each of the resting sensations. Immediately after running $500 \mathrm{~m}$ at $70 \%$ (self determined) pace, they marked the scale for the sensations experienced during activity. The observer timed each run to ensure it was within five seconds of the time achieved at first testing.

\section{Needling protocol}

All needling was performed by the same investigator (PB). Trigger points in the gluteals were isolated by palpation, ensuring reproduction of symptoms. Most of these were in the region of the upper outer buttock quadrant, and subjects had three to five points each. Therapeutic needling was performed with $0.30 \mathrm{~mm}$ diameter, $25 \mathrm{~mm}$ long sterile acupuncture needles (Seirin Corp, Shizuoka, Japan). Each needle is individually packed. The needle pierced the skin and was advanced into the trigger point. Reproduction of recognisable pain or visualisation of a local twitch response indicated appropriate placement. ${ }^{2}$ The needle was then partially withdrawn and repeatedly advanced into the muscle until the pain resolved and no further twitches were seen. This usually corresponded to one minute of treatment for 
each point. Placebo needles were a modification of the acupuncture needles. Needles were unwrapped, the sharp tip removed, and the needle glued back in its sheath. It was dipped in Betadine, but not resterilised, as it did not pierce the skin. Subjects did not see the needles at any stage. These needles made the same sound as real needles when released from their sheaths. Placebo needling involved applying the tip of a blunted needle to the skin over the trigger point. Pressure was applied for 10 or more seconds and the needle moved slightly to simulate real needling. This was repeated at each point for up to one minute.

\section{Randomisation}

Randomisation was performed using random number tables and the allocation was held by a third party until contacted by the intervention investigator.

\section{Blinding and prevention of unblinding}

Both subject and outcomes investigator were blinded. Placebo needles had previously been assessed for reliability in 10 volunteers. The incidence of correctly identifying placebo was no more than chance $\left(\chi^{2} 0.975, \mathrm{p}=0.323\right)$. Subjects remained prone during treatment, and did not discuss the treatment or its effects subsequently. Blinding was assessed immediately after treatment and at trial completion, and results were sealed until data were collated.

\section{Contamination and cointerventions}

Subjects were requested not to have treatment to their trigger points for one week before the study and no other manual treatment on their back or lower limbs for the three days of the study. They continued playing and training as usual.

\section{Statistical analysis}

Statistical analysis was performed using the Statistical Package for the Social Sciences (SPSS; Norusis/SPSS Inc, Chicago, Illinois, USA). The level of significance was set at 0.05 for all tests unless otherwise specified.

Before analysis, data were examined to identify potential violation of statistical assumptions. Normally distributed data were analysed with parametric statistics, and nonnormal data with non-parametric statistics.

The placebo and real needling group were examined for comparability with respect to height, weight, SLR, hip IR, and VAS scores. Independent $t$ tests or Kruskall-Wallis tests were used as appropriate.

The effects of the intervention were compared in each group over the four time points using a repeated measures analysis of variance.

A Friedman test was performed to evaluate the effects of both interventions on VAS over time. Significant results were further analysed with a Wilcoxon signed ranks test. MannWhitney U testing showed any differences attributable to group allocation. Owing to the number of comparisons performed, a more conservative $\alpha$ level of 0.013 was used to protect against type I error. ${ }^{46}$

VAS data is presented as box plots. The median is represented by a line in the box, and the limits of the box are the interquartile distance. The whiskers extend to the minimum and maximum values, and outliers are represented by small circles.

Global rating of change scores (a five point scale) were dichotomised in the following manner: 1 , scores of 4 or 5 that is, better and much better (improvement); 2 , scores of 1 , 2 , or 3-that is, much worse, worse, and no change (no improvement). $\chi^{2}$ analyses with standardised residuals were performed to test for associations between group allocation and perceived improvement. $\chi^{2}$ analysis was used to assess the effectiveness of subject and investigator blinding immediately after the intervention and on completion of the trial.

\section{RESULTS}

Eighty five subjects were screened; 60 were included, but one was subsequently excluded for an adductor magnus cyst on MRI. Fifty nine subjects completed the measures before and immediately after the intervention. Fifty eight completed the measures at 24 hours, and 52 completed the measures at 72 hours. Drop outs were due to outside commitments, but subjects all completed blinding and global change surveys by telephone.

\section{Baseline characteristics}

There was no significant difference between the groups before the intervention for age, height, weight, SLR, or hip IR. VAS measures were equivalent for all but resting gluteal pain, which was significantly higher in the therapeutic needling group.

\section{Range of motion}

All results were analysed with carry forward of the last result, and exclusion of missing data points and outcomes were similar. Results are reported for the carry forward analysis only. Table 2 gives the results for SLR to P1, SLR to P2, and hip IR. There was no significant change in range of motion in either group.

\section{VAS}

There was no significant change in VAS scores for any of the resting variables or for gluteal pain after running. Both groups showed significant improvement in hamstring tightness $(\mathrm{p}<0.001)$, hamstring pain $(\mathrm{p}<0.001)$, and gluteal tightness $(\mathrm{p}=0.001$ for therapeutic and $\mathrm{p}<0.001$ for placebo) after running. Figure 4 gives the results of the running scores. The improvements occurred immediately after the intervention, and there was no significant change in subsequent measures.

There was no significant difference in the magnitude of the change related to group allocation $(\mathrm{p}>0.013)$. On global rating of change analysis, no subjects felt worse after treatment. Fourteen subjects in the placebo and 18 subjects in the therapeutic group felt that they had improved, and one subject in the therapeutic group felt much improved. This did not translate to a significant difference when analysed statistically.

\section{Analysis of blinding}

$\chi^{2}$ analysis revealed that the therapeutic group correctly identified allocation immediately after treatment $(\mathrm{p}<0.001)$, but no association at the end of the study $(p=0.062)$.

There was a significant association between observer opinion and group allocation $(\mathrm{p}=0.043)$, with values trending towards more correct opinions in the therapeutic group.

\section{Adverse outcomes}

Two subjects experienced syncopal responses to the needling, recovered quickly, and completed the study. One subject experienced atypical chest pain in the initial run. After full medical evaluation, he started the trial again two days later.

\section{DISCUSSION}

The lack of change in SLR and hip IR after treatment in this research may indicate one of three things. Firstly, the postulated restriction in range of motion may not be associated with symptoms. This research does not answer this question, as participants often had bilateral symptoms, 
pathology in the contralateral hamstring, or injuries to other muscles of the contralateral limb involved in pelvic movement. Secondly, these may be appropriate outcome measures that have indicated no change in muscle length as a result of dry needling, or, thirdly, the outcome measures used may be inappropriate to recognise any change. Regardless of the reason, it can be said that SLR and hip IR are not suitable reassessment measures after treatment of gluteal trigger points.

Self reports of improvement have been shown to be equivalent for placebo and therapeutic needling and are most appropriate for assessing response to treatment.

Most placebo controlled studies on needling therapy of trigger points have obtained similarly negative results with respect to range of motion and other objective measures, while still reporting an improvement in pain similar to that seen in this study. ${ }^{36}{ }^{37}$ The effective placebo interventions have all involved the application of a stimulus strong enough to be confused with needle penetration. Similar results are seen with the use of other counter stimulatory interventions, but are not reproduced in the absence of any noxious input.

The mechanism of the pain relief seen with trigger point treatments is not known. Early theories suggested that direct stimulation of the ischaemic tight muscle segment may allow it to relax and may alter local mediator and neurotransmitter concentrations, thereby reducing the pain signals. ${ }^{2}$ More recently, diffuse noxious inhibitory control, a central increase in opioid release in response to a painful stimulus, resulting in modulation of pain transmission, has been implicated in the analgesia of acupuncture and the prolonged pain relief described with local anaesthetics. ${ }^{41}$ Opioid blockade results in return of trigger point pain after effective treatment. ${ }^{47}$ This system has also been implicated in the production of a runner's high ${ }^{48}$ and the placebo effect. ${ }^{47}$

Therefore, despite the current lack of evidence to support objective improvements in muscle length or function after dry needling, there is now a convincing amount of data to suggest that significant subjective improvements are seen with a number of forms of counter stimulation. Although often discredited as being due to the placebo effect, there are still central physiological mechanisms that may be responsible for a global reduction in pain perception. Indeed, these mechanisms may be responsible for effects previously disregarded as due to placebo, which can have important roles to play in achieving clinical improvements.

Certainly, there has been a long standing argument in the literature on acupuncture since the discovery that acupuncture at non-specific points can afford similar levels of pain relief to that achieved when relevant points are treated. ${ }^{49-51}$ Not only have spinal cord opioids and pain gating been implicated, but functional brain imaging has revealed changes in areas involved in pain processing after needle treatment. $^{52} 53$

It was expected that the placebo used in the current research would be less traumatic. However, the success of blinding of subjects may indicate that the stimulus was equivalent to a needle penetration. The equivalent responses seen with placebo and therapeutic interventions to date do suggest a centrally mediated analgesic effect, rather than a local muscle response.

\section{Possible reasons for observed outcomes}

An appropriate study population was identified and recruited. Numbers were calculated for $80 \%$ power assuming a $15^{\circ}$ change in SLR.

It is unusual in clinical practice to use dry needling as a single isolated treatment. We cannot comment from these results as to whether repeated treatment or combination treatment may have a different outcome.
What is already known on this topic

Many treatments for trigger points have not proved to be more effective than placebo when put to scientific analysis. In patients with chronic pain, many counter stimulatory interventions have shown equivalent improvements.

\section{What this study adds}

This is the first study on trigger points to be performed in athletes. It confirms the presence of posterior thigh pain in the absence of local muscle pathology, and emphasises the importance of using subjective outcome measures to assess clinical response, while agreeing with results from patients with chronic pain, indicating the effectiveness of counter stimulatory interventions in trigger point treatment.

\section{Clinical implications}

Both groups improved subjectively after intervention. Running discomfort was affected more than resting sensations. There was no significant increase in gluteal pain after the therapeutic intervention despite soreness having previously been described as the major side effect of this treatment. ${ }^{31}$ Although local muscle stimulation cannot be ruled out as contributing to pain relief, both the placebo effect and central modification of pain perception must be seriously entertained as possible mechanisms for this effect.

\section{Strengths and limitations}

The lack of a control group means that we cannot state that the results seen were due to the intervention and were not a reflection of the natural history of disease. However, subjects generally complain of these symptoms over months to years.

Blinding was effective, and the power was adequate to reveal VAS changes. It is unlikely that there was any change in range of motion.

\section{ACKNOWLEDGEMENTS}

We acknowledge the valuable assistance of Mark Lourenz, Trevor Allen, Ewa Stendur, Barry Stillman, and Janet McKinney in developing and performing this trial.

\section{Authors' affiliations}

L Huguenin, Australian Institute of Sport, Belconnen, ACT, Australia P D Brukner, H Wajswelner, K Bennell, Centre for Health, Exercise and Sports Medicine, School of Physiotherapy, University of Melbourne, Melbourne, Australia

P McCrory, British Journal of Sports Medicine, Centre for Sports Medicine Research and Education, Parkville, Victoria, Australia P Smith, St Vincent's Hospital, Melbourne

Patient consent has been obtained for figures 1, 2, and 3

Competing interests: none declared

\section{REFERENCES}

1 Brukner P, Khan K. Clinical sports medicine, 2nd ed. Sydney: McGraw-Hill, 2001.

2 Simons D, Travell J, Simons L. Travell \& Simons' myofascial pain and dysfunction: the trigger point manual, 2nd ed. Baltimore: Williams and Wilkins, 1998.

3 Travell J. Factors affecting pain of injection. JAMA 1955;158:368-71.

4 Mense S, Simons D. Muscle pain. Understanding its nature, diagnosis and treatment, 1st ed. Baltimore: Lippincott Williams \& Wilkins, 2001.

5 Vaeroy H, Sakurada T, Forre O, et al. Modulation of pain in fibromyalgia (fibrositis syndrome): cerebrospinal fluid (CSF) investigation of pain related neuropeptides with special reference to calcitonin gene related peptide (CGRP). J Rheumatol 1989;16(suppl 19):94-7. 
6 Henry J. Concepts of pain sensation and its modulation. J Rheumatol 1989;16(suppl 19):104-12.

7 Mense S. Biochemical pathogenesis of myofascial pain. J Musculoskel Pain 1996;1:145-62.

8 Hoheisal U, Mense S, Simons D, et al. Appearance of new receptive fields in rat dorsal horn neurons following noxious stimulation of skeletal muscle: a model for referral of muscle pain? Neurosci Lett 1993;153:9-12.

9 Melzack R. Recent concepts of pain. J Med 1982;13:147-60.

10 Hong C-Z. Pathophysiology of myofascial trigger point. J Formos Med Assoc 1996:95:93-104.

11 Bendtsen L, Jensen R, Olesen J. Qualitatively altered nociception in chronic myofascial pain. Pain 1996;65:259-64.

12 McNulty W, Gervirtz R, Hubbard D, et al. Needle electromyographic evaluation of trigger point response to a psychological stressor. Psychophysiology 1994;31:313-16.

13 Chen J-T, Chen S-M, Kuan T-S, et al. Phentolamine effect on the spontaneous electrical activity of active loci in a myofascial trigger spot of rabbit skeletal muscle. Arch Phys Med Rehabil 1998;79:789-94.

14 Toomey M. In: Zuluaga M, Briggs C, Carlisle J, et al. Sports physiotherapy: applied science and practice. Melbourne: Churchill Livingstone, 1998.

15 Bohannon R, Gadjosik R, LeVeau B. Contribution of pelvic and lower limb motion to increases in the angle of passive straight leg raising. Phys Ther 1985;65:474-6.

16 Cameron D, Bohanon R, Owen S. Influence of hip position on measurements of the straight leg raise. J Orthop Sports Phys Ther 1994;19:168-72.

17 Clark S, Christiansen A, Hellman D, et al. Effects of ipsilateral anterior thigh soft tissue stretching on passive unilateral straight leg raise. J Orthop Sports Phys Ther 1999:29:4-9.

18 Gajdosik R, Albert C, Mitman J. Influence of hamstring length on the standing position and flexion range of motion of the pelvic angle, lumbar angle and thoracic angle. J Orthop Sports Phys Ther 1994;20:213-19.

19 Hall T, Hepburn M, RL E. The effect on lumbosacral posture on a modification of the straight leg raise test. Physiotherapy 1993;79:566-70.

20 Sullivan M, Dejulia J, Worrell T. Effect of pelvic positioning and stretching method on hamstring muscle flexibility. Med Sci Sports Exerc 1992;24:1383-9.

21 Graff-Redford SB, Reeves JL, Baker RL, et al. Effects of transcutaneous electrical nerve stimulation on myofascial pain and trigger point sensitivity. Pain 1989;37:1-5.

22 Hsueh TC, Cheng PT, Kuan TS, et al. The immediate effectiveness of electrical nerve stimulation and electrical muscle stimulation on myofascial trigger points. Am J Phys Med Rehabil 1997:76:471-6.

23 Gam A, Warming S, Larsen $\mathrm{LH}$, et al. Treatment of myofascial trigger points with ultrasound combined with massage and exercise: a randomised controlled trial. Pain 1998;77:73-9

24 Snyder-Mackler L, Bork C, Bourbon B, et al. Effect of helium-neon laser on musculoskeletal trigger points. Phys Ther 1986:66:1087-90.

25 Olavi A, Pekka R, Pertii K, et al. Effects of the infra red laser therapy at treated and non-treated trigger points. Acupunct Electrother Res 1989;14:9-14.

26 Ceccherelli F, Altafini L, Lo Castro G, et al. Diode laser in cervical myofascial pain: a double-blind study versus placebo. Clin J Pain 1989;5:301-4.

27 Thorsen H, Gam A, Svensson B, et al. Low level laser therapy for myofascial pain in the neck and shoulder girdle: a double-blind, cross-over study. Scand J Rheumatol 1992;21:139-41.

28 Waylonis G, Wilke S, O'Toole D, et al. Chronic myofascial pain: management by low-output helium-neon laser therapy. Arch Phys Med Rehabil 1988;69:1017-20.

29 Frost F, Jessen B, Siggard-Andersen J. A control, double blind comparison of mepivicaine injection versus saline injection for myofascial pain. Lancet 1980 Mar 8:499-500.
30 Garvey TA, Marks MR, Wiesel SW. A prospective, randomised, double blind evaluation of trigger point therapy for lower back pain. Spine 1989;14:962-4.

31 Hong C-Z. Lidocaine injection versus dry needling to myofascial trigger point. The importance of the local twitch response. Am J Phys Med Rehabil 1994; 73:256-63.

32 Ray M. Isotonic glucose solution in the treatment of fibrositis [correspondence]. BMJ 1941 Dec 13:850.

33 Souttar $\mathbf{H}$. Acute lumbago treated by the injection of quinine and urea. BMJ 1923 Nov 17:915-16.

34 Cheshire W, Abashian S, Mann J. Botulinum toxin in the treatment of myofascial pain syndrome. Pain 1994;59:65-9.

35 Wheeler A, Goolkasian P, Gretz S. A randomized, double blind, prospective pilot study of botulinum toxin injection for refractory, unilateral, cervicothoracic, paraspinal, myofascial pain syndrome. Spine 1998;23:1662-7.

36 Karakurum B, Karaalin O, Coskun O, et al. The "dry needle technique" intramuscular stimulation in tension-type headache. Cephalalgia 2001;21:813-17.

37 McMillan A, Nolan A, Kelly P. The efficacy of dry needling and procaine in the treatment of myofascial pain in the jaw muscles. J Orofac Pain 1997; 11:307-14

38 Button M. Muscular rheumatism: local injection treatment as a means to rapid restoration of function. BMJ 1940 Aug 10:183-5.

39 Howard R. The use of local anaesthesia in the relief of chronic pain. Med J Aust 1941 March 8:298-9.

40 Kelly $M$. The treatment of fibrositis and allied disorders by local anaesthesia. Med J Aust 1941 Mar 8:294-8.

41 Hameroff SR, Crago BR, Blitt CD, et al. Comparison of bupivacaine, etidocaine and saline for trigger point therapy. Anesth Anal 1981;60:752-5.

42 Byrn C, Olsson I, Falkheden L, et al. Subcutaneous sterile water injections for chronic neck and shoulder pain following whiplash injuries. Lancet 1993;341(8843):449-52.

43 Streitberger K, Kleinhenz J. Introducing a placebo needle into acupuncture research. Lancet 1998;352:364-5.

44 Pomeranz S, Heidt R. MR Imaging in the prognostication of hamstring injury. Radiology 1993; 189:897-900.

45 Bennell K, Khan K, Matthews B, et al. Hip and ankle range of motion and hip muscle strength in young novice female ballet dancers and controls. Br J Sports Med 1999:33:340-6.

46 Portney LG, Watkins MP. Foundations of clinical research: applications to practice, 2nd ed. Upper Saddle River, NJ: Prentice Hall, 2000.

47 Fine PG, Milano R, Hare BD. The effects of myofascial trigger point injections are naloxone reversible. Pain 1988;32:15-20.

48 Koltyn K. Exercise-induced hypoalgesia and intensity of exercise. Sports Med 2002;32:477-87

49 Gaw A, Chang L, Shaw L-C. Efficacy of acupuncture on oseoarthritic pain. N Engl J Med 1975;293:375-8.

50 Birch S, Jamison R. Controlled trial of Japanese acupuncture for chronic myofascial neck pain: assessment of specific and nonspecific effects of treatment. Clin J Pain 1998;14:248-55.

51 Lee $\mathbf{P}$, Andersen T, Modell J, et al. Treatment of chronic pain with acupuncture. JAMA 1975;232:1133-5.

52 Wu M-T, Hsieh J-C, Xiong J, et al. Central nervous pathway for acupuncture stimulation:localisation of processing with functional MR imaging of the brain: preliminary experience. Radiology 1999;212:133-41.

53 Bialla G, Sotgiu M, Pellegata G, et al. Acupuncture produces central activations in pain regions. Neuroimage 2001;14:60-6. 